\title{
Spontaneous Tumor Lysis Syndrome Presenting Acute Kidney Injury with Extreme Hyperuricemia and Urinary Stone: A Rare Case of Spontaneous Tumor Lysis Syndrome
}

\author{
Seong Heon Kim, M.D. \\ Eu Jeen Yang, M.D. \\ Young Tak Lim, M.D., Ph.D. \\ Su Young Kim, M.D., Ph.D. \\ Department of Pediatrics, Pusan \\ National University Children's Hospital, \\ Yangsan, Korea \\ Corresponding author: \\ Su Young Kim, M.D., Ph.D. \\ Department of Pediatrics, Pusan National \\ University Children's Hospital, 20 Geumo- \\ ro, Mulgeum-eup, Yangsan, Goungnam \\ 50612 , Korea \\ Tel: +82-55-360-3151 \\ Fax: +82-55-360-2181 \\ E-mail:suyung@pusan.ac.kr \\ Received: 13 February 2017 \\ Revised: 23 March 2017 \\ Accepted: 24 March 2017
}

Tumor lysis syndrome is a serious complication of malignancy, resulting from the massive and rapid release of cellular components into the blood. Generally, it occurs after initiation of chemotherapy. The onset of spontaneous tumor lysis syndrome (STLS) before anti-cancer treatment is rare and occurs mostly in Burkitt lymphoma and non-Hodgkin's lymphoma. There are only a few case reports in children. Here, we report a case of STLS secondary to T-cell acute lymphoblastic leukemia (ALL), which presented with urinary stone and subsequent acute kidney injury with severe hyperuricemia. Occult malignancy should be considered in case of unexplained acute kidney injury with extreme hyperuricemia.

Key words: Acute kidney injury, Hyperuricemia, Spontaneous tumor lysis syndrome, Renal stone

\section{Introduction}

Tumor lysis syndrome is the most common disease related emergency in hematologic malignant patients which occurs when tumor cells release their several contents into blood typically in response to chemotherapy ${ }^{1)}$. Characteristic findings are hyperuricemia, hyperkalemia, hyperphosphatemia and hypocalcemia. Furthermore these disturbances can progress to renal insufficiency, cardiac arrhythmia, seizure and death ${ }^{1)}$.

Tumor lysis syndrome can occur before initiation of anticancer treatment which named spontaneous tumor lysis syndrome (STLS). STLS is rare, especially in the absence of other signs of malignancy such as hyperleukocytosis or mass lesion ${ }^{2}$. STLS develops mostly in Burkitt lymphoma and non-Hodgkin lymphoma which has an extraordinarily rapid cell turnover rate ${ }^{3)}$. But the reason of this phenomenon remains uncertain.

There are only a few case reports in children with STLS which mostly were secondary to Burkitt lymphoma and non-Hodgkin lymphoma ${ }^{3,4)}$. STLS with acute kidney injury (AKI) is very uncommon initial presentation of leukemia and STLS presenting with renal stone is extremely rare. To our knowledge, only two cases of STLS secondary to T cell acute lymphoblastic leukemia (ALL) were reported under 6 years age group and only one case with renal calculi as presenting symptom in children ${ }^{2,5}$. 


\section{Case report}

A 6-year-old boy was referred to our hospital for generalized tonic clonic seizure. One month ago, he visited other hospital for episodic right-sided flank pain and evaluations revealed gross hematuria, hyperuricemia and urinary stone on right uretero-vecical junction (UVJ). No specific treatment was done except adequate oral hydration. For 15 days before admission, he would not eat well and his activity gradually decreased with several episode of vomiting.

His blood pressure was $160 / 90 \mathrm{mmHg}$, pulse rate 100 beats $/ \mathrm{min}$ and respiratory rate $20 / \mathrm{min}$. He did not have any dysmorphic features and he had developed normally. Physical examination showed tender hepatomegaly $(2 \mathrm{~cm}$ below costal margin) without splenomegaly.

There was no significant change on weight and his volume status appeared to be normal by physical examination, but severe hypertension could mean intravascular volume overload. On the way to our hospital, his seizure stopped but he appeared acutely ill and drowsy. His initial laboratory findings were shown in table 1 . There was no blast on his peripheral blood smear test and urinalysis showed significant uric acid crystal and microscopic hematuria of 10-15 $\mathrm{RBC}$ s per high power field. Initial urine $\mathrm{pH}$ was 5.5 and uric acid excretion per GFR was $6.26 \%$ during AKI and 0.78 $\%$ after renal function recovered which meant uric acid over excretion. Ultrasonography of both kidneys showed increased cortical echogenicity, slightly loss of corticomedullary differentiation, tiny hyperehoic dots in medulla of both enlarged kidney $(14.3 \mathrm{~cm}$ and $12.9 \mathrm{~cm})$ and non-visible right UVJ stone, which was thought to pass out (Fig. 1). Suspecting hypertensive encephalopathy due to AKI or uremic encephalopathy, we started emergent hemodialysis. His renal function, blood pressure, uric acid and electrolyte abnormalities gradually improved with appropriate therapy after 2 times of hemodialysis. But serum uric acid level increased again (from $6.1 \mathrm{mg} / \mathrm{dL}$ to $12.9 \mathrm{mg} / \mathrm{dL}$ ) soon after hemodialysis, so allopurinol treatment was added on. The cause of AKI was unclear and after literature review, we suspected that AKI presumed to be secondary to acute uric acid nephropathy caused by STLS because of extreme hyperuricemia up to $55.2 \mathrm{mg} / \mathrm{dL}(2.5-8.0)$ and hepatomegaly. Treatment and disease course of our patient is shown in Fig. 2.
On hospital day \#7, we performed bone marrow biopsy which demonstrated normocellular marrow without evidence of malignancy. On hospital day \#15, abdominal pain with hepatomegaly aggravated and uncontrolled high fever occurred. For 1 week, serum levels of uric acid, BUN, creatinine and phosphate were also getting increased and thrombocytopenia worsened (Table 1). Twenty percent of blasts were seen on his peripheral blood smear and subsequent second bone marrow biopsy demonstrated $\mathrm{T}$ cell ALL (Fig. 2). Then, he was referred to division of pediatric hemato-oncology on our hospital and induction chemotherapy started. On initiation of chemotherapy, intermittent hemodilaysis was performed again due to tumor lysis syndrome. After 1 week of chemotherapy, serum uric acid began to stay within reference range. Final diagnosis of STLS secondary to T cell ALL presenting with extreme hyperuricemia, renal stone and AKI was made.

\section{Discussion}

Tumor lysis syndrome is a metabolic derangement characterized by hyperuricemia, hyperkalemia, hyperphosphatemia, hypocalcemia and AKI. It is a medical emergency and typically occurs after chemotherapy. Risk

\begin{tabular}{lrrrr}
\multicolumn{5}{l}{ Table 1. Details in Laboratory Test } \\
\hline Laboratory tests & Day 1 & Day 7 & Day 15 & Day 30 \\
\hline Hb, g/dL & 9.9 & 9.8 & 8.8 & 11.5 \\
WBC, 10 $/ \mathrm{lL}$ & 11.9 & 5.76 & 7.94 & 2.52 \\
Blast, \% & 0 & 0 & 20 & 0 \\
Platelet, $10^{3} / \mathrm{uL}$ & 242 & 196 & 65 & 158 \\
AST, IU/L & 17 & 80 & 42 & 13 \\
ALT, IU/L & 4 & 80 & 23 & 28 \\
ALP, IU/L & 308 & 332 & 459 & 334 \\
LD H, IU/L & 460 & 419 & 1,593 & 394 \\
bilirubin, mg/dL & $<0.1$ & 0.3 & 0.7 & 1.4 \\
Albumin, g/d L & 4.1 & 3.5 & 4.0 & 3.6 \\
Uric acid, mg/dL & 47.6 & 4.1 & 8.9 & 4.5 \\
BUN, mg/dL & 119.3 & 14.0 & 21.0 & 30 \\
Cr, mg/dL & 4.77 & 0.6 & 2.1 & 0.5 \\
Na, mmol/L & 127 & 140 & 135 & 133 \\
K, mmol/L & 3.8 & 3.8 & 4.9 & 4.8 \\
Cl, mmol/L & 96 & 104 & 101 & 105 \\
Ca, mg/dL & 9.2 & 7.2 & 8.8 & 9.0 \\
P, mg/dL & 7.4 & 4.2 & 5.4 & 3.5 \\
\hline
\end{tabular}




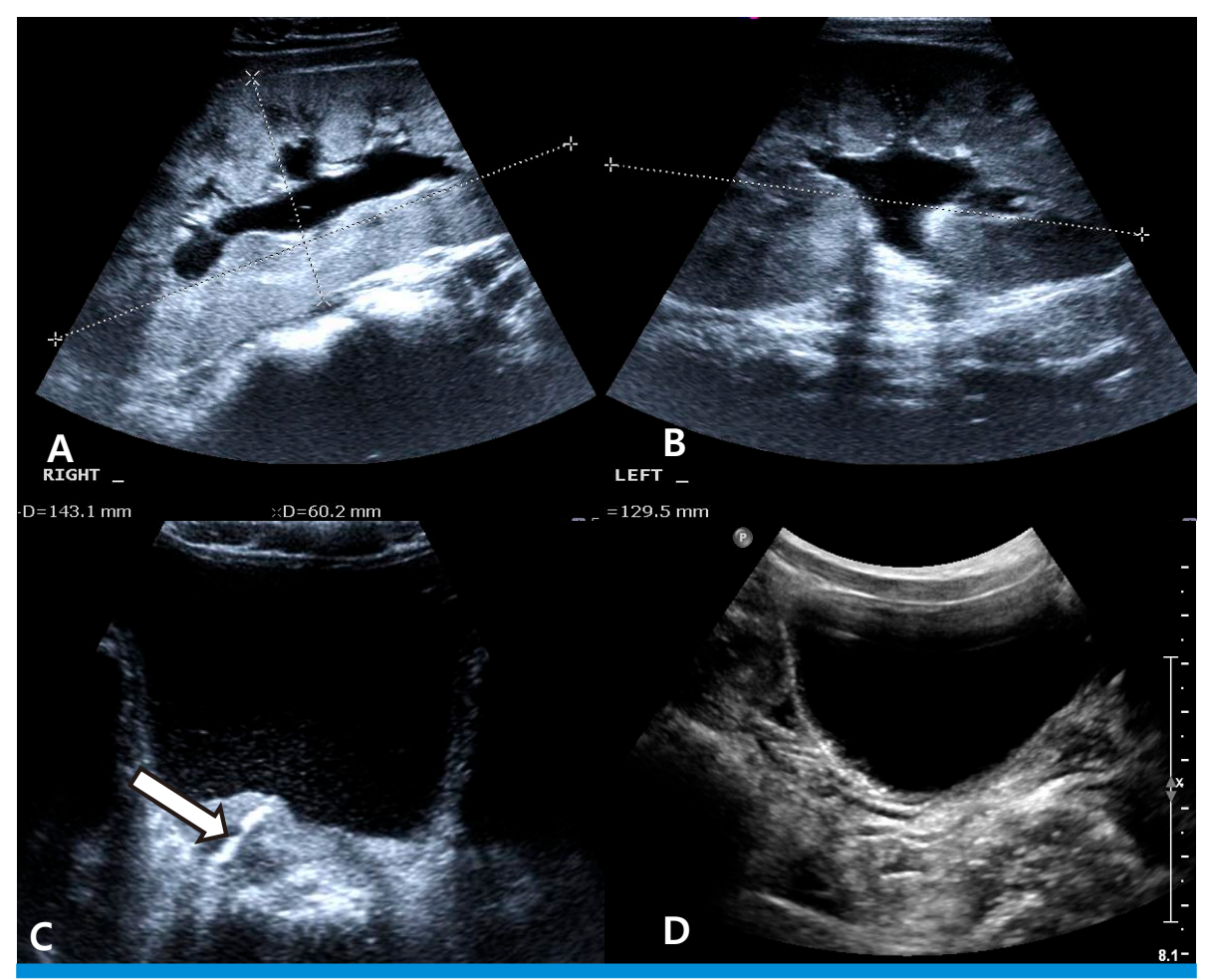

Fig. 1. Ultrasonograpy of the kidney and bladder. Initial ultrasonograpy at other hospital revealed markedly enlarged both kidney (RK $14.3 \mathrm{~cm}$, LK $12.9 \mathrm{~cm}$ ) with increased cortical echogenicity and hydronephrosis (A, B). White arrow (C) showed Rt UVJ stone with swelling, but we could not find the UVJ stone on follow-up US in our hospital(D). It is suspected that the stone could spontaneously pass out.

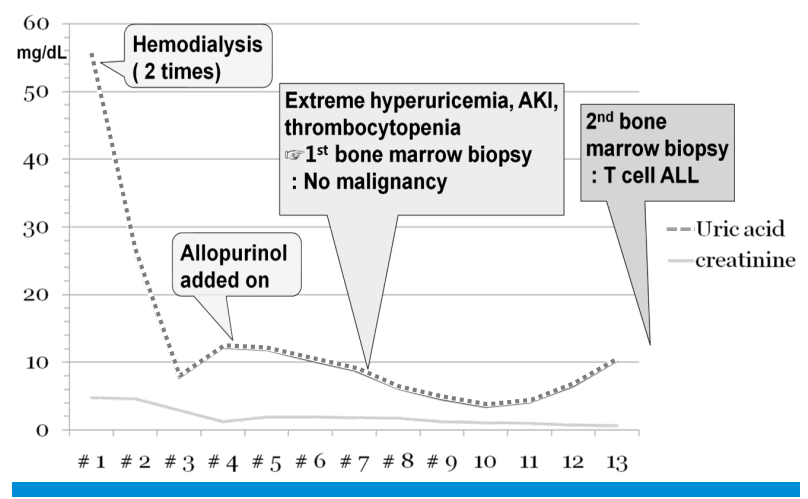

Fig. 2. Treatment and disease course of the patient.

factors for the development of TLS are large tumor burden, extensive metastasis, renal infiltration and high rate of cell turnover etc ${ }^{1)}$.

STLS can occur mostly in Burkitt lymphoma and nonHodgkin lymphoma which has large tumor burden, although it is rare. Hsu et al reported 12 cases of STLS in a review of about 1,000 adult patients with AKI which consisted of 4 with Burkitt lymphoma, 6 with non-Hodgkin lymphoma and one with B cell ALL ${ }^{4}$. Cases of STLS due to T cell ALL were more scarce, especially in pediatric patients. Only two cases of STLS secondary to T cell ALL were reported under 6 years age group ${ }^{2,5)}$. Both of two cases commonly showed acute kidney injury with extreme hyperuricemia (>24 mg/dL). STLS with AKI is very uncommon initial presentation of leukemia and STLS presenting with renal stone is extremely rare. Recent case by Daly demonstrated renal calculi as presenting symptom which was finally diagnosed with uric acid nephrolithiasis and AKI secondary to STLS ${ }^{5}$. Our case also presented nephrolithiasis on UVJ at first and after that diagnosed with same disease. So our case is the second to describe gross hematuria and nephrolithiasis from STLS in the pediatric population.

The reason of this spontaneous tumor response without anticancer therapy remains unclear. Some reports suggested that potential causes of STLS include endogeneous glucocorticoid with infection and fever, but our patient did not experience fever ${ }^{6,7}$. Further studies are needed to find out the reason. 
Hyperuricemia in tumor lysis syndrome results from the catabolism of nucleic acids to hypoxanthine, xanthine and then uric acid by action of xanthine oxidase. Uric acid can induce AKI not only by intrarenal crystallization but also by crystal independent mechanism, such as renal vasoconstriction, impaired autoregulation, decreased renal blood flow, oxidation and inflammation ${ }^{1)}$. Treatment options for this conditions include massive hydration, the xanthine oxidase inhibitor or the recombinant urate oxidase and hemodialysis ${ }^{8}$. In a setting of confirmed malignancy, most clinicians are able to consider the possibility of tumor lysis syndrome when starting chemotherapy. But in the absence of sign of malignancy, it is quite difficult to consider the possibility of STLS. Our patient showed marked hyperuricemia in which serum level of uric acid increased upto $55.2 \mathrm{mg} / \mathrm{dL}$ (2.5-8.0). Uric acid excretion per GFR was $6.26 \%$ during AKI and $0.78 \%$ after renal function recovered which meant uric acid over excretion (reference, 0.23-0.45). In previously reported two cases, uric acid levels were also very high as with our patient (30 $\mathrm{mg} / \mathrm{dL},>24 \mathrm{mg} / \mathrm{dL})^{2,5)}$. AKI with extreme hyperuricemia can be a first manifestation of spontaneous tumor lysis syndrome and clinicians should consider occult malignancy and STLS.

The pathophysiology of AKI in STLS is known to be multifactorial; volume depletion and uric acid nephropathy ${ }^{9}$. Futhermore, hematologic malignancy itself could involve kidney and contribute to AKI. Schlick K et al. reported a case of adult STLS patient with marked enlarged kidney which was finally confirmed to T cell ALL by performing renal biopsy ${ }^{10)}$. Our patient had also bilateral enlarged kidney for its age and we could not conclude that AKI in our case is due to solely STLS because we did not performed renal biopsy and renal involvement of T cell ALL may involve in this process although it is very rare.

In conclusion, in a clinical situation of marked hyperuricemia with acute kidney injury, we need to consider occult malignancy and STLS.

\section{Conflict of interest}

No potential conflict of interest relevant to this was reported.

\section{Acknowledgments}

This research was supported by 2 years grant from Pusan National University.

\section{References}

1. Howard SC, Jones DP, Pui CH. The tumor lysis syndrome. N Engl J Med 2011;364:1844-54.

2. Kobayashi D, Wofford MM, McLean TW, Lin JJ. Spontaneous tumor lysis syndrome in a child with T-cell acute lymphoblastic leukemia. Pediatr Blood Cancer 2010;54:773-5.

3. Cohen LF, Balow JE, Magrath IT, Poplack DG, Ziegler JL. Acute tumor lysis syndrome. A review of 37 patients with Burkitt's lymphoma. Am J Med 1980;68:486-91.

4. Hsu HH, Chan YL, Huang CC. Acute spontaneous tumor lysis presenting with hyperuricemic acute renal failure: clinical features and therapeutic approach. J Nephrol 2004;17:50-6.

5. Daly GF, Barnard EBG, Thoreson L. Renal Calculi: An Unusual Presentation of T-Cell Acute Lymphoblastic Leukemia. Pediatrics 2016;137.

6. Chen RL, Chuang SS. Transient spontaneous remission after tumor lysis syndrome triggered by a severe pulmonary infection in an adolescent boy with acute lymphoblastic leukemia. J Pediatr Hematol Oncol 2009;31:76-9.

7. Lotfi M, Brandwein JM. Spontaneous acute tumor lysis syndrome in acute myeloid leukemia? A single case report with discussion of the literature. Leuk Lymphoma 1998;29:625-8.

8. Davidson MB, Thakkar S, Hix JK, Bhandarkar ND, Wong A, Schreiber MJ. Pathophysiology, clinical consequences, and treatment of tumor lysis syndrome. Am J Med 2004;116:546-54.

9. Wilson FP, Berns JS. Onco-nephrology: tumor lysis syndrome. Clin J Am Soc Nephrol 2012;7:1730-9.

10. Schlick K, Schilcher G, Krisper P, Neumeister P, Linkesch W, BehamSchmid C, et al. Spontaneous tumor lysis syndrome presenting as acute renal failure in an adult with de novo aleukemic T-ALL: value of renal biopsy? J Clin Oncol 2011;29:e494-6. 